\title{
Coronary-Coronary bypass graft as a safe and an alternative technique for revascularization of coronary arteries
}

\begin{abstract}
:
Coronary-coronary bypass graft was first performed by Rowland and Grooters. This technique can be performed between two segments of the same coronary artery using saphenous vein grafts or free arterial grafts in on/off-pump coronary artery bypass grafting, also can be an alternative safe technique in some cases as calcified ascending aorta, porcelain aorta and insufficient graft length. The coronarycoronary bypass graft can provide nearly the same flow rate as conventional coronary artery bypass graft, another advantage this technique is that we can use to decrease sternal and respiratory morbidity. we performed this technique to a 55-year-old woman to whom coronary angiography showed critical three-vessel disease, using a free segment of right internal mammary artery, combined to conventional coronary artery bypass graft. The postoperative course was uneventful with the absence of ischemic lesions and the grafts were patent at 6 months after procedure.
\end{abstract}

Aim: This aim of this article is to describe this technique and suggest the ways that we can use it.

Keywords: CCBG: Coronary-Coronary Bypass Graft • Myocardial Revascularization

\section{Introduction}

Coronary-coronary bypass graft was first performed by Rowland and Grooters, this technique can be performed between two segments of the same coronary artery using saphenous vein grafts or free arterial grafts in on/off-pump coronary artery bypass grafting.

\section{Technique and Clinical Summary}

The procedure was carried out by undergoing coronary artery bypass surgery, using both internal mammary arteries, and a free segment of right internal mammary artery as coronary-coronary artery bypass, to a 55-year-old woman with history of type II diabetes mellitus, hypertension and cigarette smoking, who presented a class III Canadian Cardiovascular Society angina with a non-ST elevation myocardial infarction, and to whom the coronary angiography showed critical three-vessel disease; $60 \%$ stenosis occlusion of the proximal left anterior descending artery, $90 \%$ occlusion of the diagonal branch, $90 \%$ occlusion of the mid the left circumflex, and $90 \%$ occlusion of its the third segment .While the echocardiography showed a preserved fraction ejection at $60 \%$, and homogeneous kinetics. The duplex ultrasound of the supra-aortic trunks was normal.

\section{Technique description}

A median sternotomy incision is used, both internal mammary arteries are harvested using skeletonized technique; the left internal mammary artery is harvested proximally from above the left subclavian vein to its bifurcation. The right internal mammary artery is harvested proximally from
Bzikha Reda ${ }^{12^{*}}$, Gautier Charles Henri ${ }^{1,2}$

'Department of Cardiovascular Surgery, Université Paris Descartes, Sorbonne Paris Cité, Paris, 75006, France ${ }^{2}$ Department of Cardiovascular Surgery, APHP, European Georges Pompidou Hospital, Paris,75015, France

*Author for correspondence: Bzikha Reda, Department of Cardiovascular Surgery, Université Paris Descartes, Sorbonne Paris Cité, Paris, 75006, France, E-mail: redabzikha@gmail.com

Received date: November 17, 2020 Accepted date: December 01, 2020 Published date: December 08, 2020 
above the right subclavian vein to its bifurcation, after systemic heparinization the right internal mammary artery is divided distally and proximally, thus the left internal mammary artery is divided distally and then both arteries sprayed with papaverine solution. Pericardiotomy, inspection of the heart and localization of the sites of anastomosis in coronary arteries Construction of the " $T$ " graft, then cardiopulmonary bypass was achieved with aortic and twostage right arterial venous cannulation. Anterograde cardioplegia solution used after the ascending aorta cross-clamping.

Then the anastomosis was made: an end to end anastomosis of the left internal mammary graft in the second segment of the left anterior descending artery, a sequential anastomosis of the left internal mammary graft to the first diagonal artery, a side to end anastomosis at the bifurcation of the left marginal artery using the right internal mammary graft. As we can't achieve the third segment of the right coronary using the right internal mammary, we decide to proceed aright coronary-coronary artery bypass using a free segment of the right internal mammary graft, we used a proximal end-to-end anastomosis between the second segment of the right coronary artery, and the free segment of the right internal mammary graft, and the distal end-to-end anastomosis is performed at the origin of the posterior left ventricular branch.

Each artery anastomosis was made with a single continuous 8-0 Prolene suture and using optical magnification.

The patient successfully weaned from cardiopulmonary bypass after spontaneous cardiac contractility resumed. The cardiopulmonary bypass time was 118 minutes and the aortic cross-clamping was 90 minutes. The postoperative course was uneventful with the absence of ischemic lesions and the grafts were patent at 6 months after procedure.

\section{Discussion}

Coronary-coronary bypass graft was first performed by Rowland and Grooters, this technique can be performed between two segments of the same coronary artery using saphenous vein grafts or free arterial grafts in on/off-pump coronary artery bypass grafting [1]. This technique is usually used for the revascularization of the right coronary artery, but also can be used for revascularization of the left ascending artery [2]. The coronary-coronary bypass grafting is an alternative safe technique that could be used in, calcified ascending aorta, porcelain aorta, stenosis or occlusion of the subclavian artery, insufficient graft length, and harvestrelated injury of the internal mammary arteries [3]. And therefore the Stroke by atheroembolism as one of morbid complication in cardiac surgery in manipulating the diseased aorta can have prevented [2].
This technique has the physiologic advantage when the proximal anastomosis is made close to the right coronary ostium, leading to optimizing the filling and the flow of the graft, and it is more physiological when anastomosis is made from the right coronary artery to another site of its segment [4]. And so more the coronary-coronary bypass graft can provide nearly the same flow rate as conventional coronary artery bypass graft [2]. Another advantage this technique is that we can use to decrease sternal and respiratory morbidity in at risk patients, by using a single internal mammary artery for the revascularization of two different vessels and/or the revascularization of multiple lesions of various arteries [5]. For some authors the short-term, the midterm and the long term of angiograms showed patent arterial coronary-coronary conduits, and for some others the follow up of the patients showed no progression of atherosclerosis on grafted right coronary artery at the site of proximal coronary-coronary conduit anastomosis [6]. This technique may be used as a safe alternative technique for revascularization of coronary arteries.

\section{Conclusion}

CABG is often indicated when coronary arteries have a 50 to 99 percent obstruction. The obstruction being bypassed is typically due to arteriosclerosis, atherosclerosis, or both. Arteriosclerosis is characterized by thickening, loss of elasticity, and calcification of the arterial wall, most often resulting in a generalized narrowing in the affected coronary artery.

\section{References}

1. Erdil N, Ates S, Demirkilic U, Tatar H, Sag C. Coronary-coronary bypass using vein graft on a beating heart in a patient with porcelain aorta. Texas Heart Inst J. 29(1): 54-5 (2002).

2. Korkmaz AA, Onan B, Tamtekin B, et al. Right coronary revascularization by coronary-coronary bypass with a segment of internal thoracic artery. Texas Heart Inst J. 34(2): 170-4 (2007).

3. Rowland PE, Grooters RK. Coronary-coronary artery bypass: An alternative. Ann Thorac Surg 43(3): 326-8 (1987).

4. Aazami MH. The difference is meaningful: Anatomic coronary-coronary bypass or physiologic coronary-coronary bypass? J Thorac Cardiovasc Surg. 128: 799-800 (2004).

5. Bedi HS, Sharma VK, Kler TS, et al. Coronary-to-coronary bypass using a free internal mammary artery: An alternative. Ann Thorac Surg 59: 757-9 (1995).

6. Barboso G, Rusticali F. Proximal internal mammary in situ graft and distal coronary-coronary graft. Texas Heart Inst J. 27(1): 70-11 (2000). 